\title{
DERECHO Y POLÍTICAS AMBIENTALES EN LA REGIÓN DE MURCIA
}

\author{
ELISA PÉREZ DE LOS COBOS HERNÁNDEZ \\ Profesora asociada de Derecho Administrativo \\ Universidad de Murcia \\ Abogada \\ SANTIAgo M. Álvarez CARREÑo \\ Profesor titular de Derecho Administrativo \\ Universidad de Murcia
}


Sumario: 1. Breve reflexión introductoria. 2. Manifestaciones de la política y legislación ambiental de la Administración regional en Murcia. 2.1. Huella de carbono y cambio climático. 2.2. Zonas de especial protección para las aves (ZEPA). A. Lagunas de las Moreras y lagunas de Campotéjar. B. Los saladares del Guadalentín. 2.3. Estadística. 2.4. Biodiversidad. 2.5. Ganadería. 2.6. Agricultura. A. Vid y cítricos. B. Almendros, cerezos, frutales con hueso, olivos y perales. C. Derechos de régimen de pago básico y pagos directos a la agricultura y a la ganadería y otros regímenes de ayuda. 2.7. Urbanismo. 3. Ordenanzas locales. 3.1. Alcantarilla: aprobación definitiva de la Ordenanza Municipal de Calidad Odorífera del Aire (BORM, núm. 93, de 24 de abril de 2015). 3.2. Mazarrón: aprobación inicial de la modificación de la Ordenanza de Uso y Aprovechamiento de Playas en Mazarrón (BORM, núm. 65, de 20 de marzo de 2015). 3.3. Lorquí: aprobación inicial de la Ordenanza reguladora del Uso y Vertidos a la Red de Alcantarillado del Municipio de Lorquí (BORM, núm. 67, de 23 de marzo de 2015)

\section{Breve reflexión introductoria}

Este primer semestre del 2015, período al que corresponde la presente crónica, recoge la producción normativa ambiental, en sentido amplio, de la CARM. Durante estos meses, un gobierno profundamente marcado por su carácter interino y de transición no ha hecho sino mantener el parsimonioso funcionamiento de la rueda administrativa regional, lo que, en materia ambiental, se ha traducido en la aparición de normas de escaso rango normativo.

Sin embargo, la cercanía de la convocatoria electoral y, seguramente, la necesidad de granjearse los apoyos empresariales necesarios para engrasar la maquinaria partidista han determinado la rauda y sorpresiva aprobación de una nueva Ley del Suelo regional que retoma una práctica habitual en materia urbanística: facilitar los desarrollos inmobiliarios aun cuando con ellos se pongan en peligro bienes de alto contenido ambiental. Algunas de las más discutibles soluciones adoptadas por esta norma "surgida de la nada" son objeto de análisis en el apartado correspondiente de esta forzosamente breve crónica.

Agradecemos al esforzado lector que nos acompañe en esta breve panorámica de la producción normativa ambiental cuando ya se sienten en la Región de Murcia los rigores estivales.

\section{Manifestaciones de la política y legislación ambiental de la Administración regional en Murcia}

\subsection{Huella de carbono y cambio climático}

El Plan Estratégico de la Región de Murcia 2014-2020 integra, entre sus líneas de trabajo, el cambio climático y el desarrollo de la huella de carbono entre las empresas. 
Asimismo, promueve la adaptación al cambio climático entre los sectores vulnerables clave o el fomento de tecnologías eficientes que avancen en la consecución de una economía baja en carbono. Las emisiones totales de gases de efecto invernadero en la Región de Murcia se sitúan en torno a 10 millones de toneladas. Las empresas que han de participar obligatoriamente en el régimen de comercio de derechos de emisión - los sectores regulados - representan solo el $40 \%$ del total de las emisiones. Los sectores y las empresas no afectados por la obligación de participar en el comercio de derechos de emisión - sectores difusos - representan el $60 \%$ de las emisiones totales ${ }^{1}$. Para estos sectores, la Unión Europea fija para 2020 un objetivo de reducción del 10\% respecto a 2005.

En el sector empresarial, el cálculo de la huella de carbono permite comunicar los esfuerzos voluntarios en responsabilidad ambiental, así como fomentar la ecoeficiencia y la comparación de productos, servicios y procesos o benchmarking empresarial. Se trata de un elemento diferenciador de las organizaciones que deciden comprometerse con el medio ambiente y apuestan por mostrar una responsabilidad social corporativa aplicada a la lucha contra el cambio climático.

Para determinar la huella de carbono, son muchos los estándares y las normas existentes a nivel internacional. Algunas de ellas editadas en español por la Asociación Española de Normalización y Certificación (AENOR) ${ }^{2}$. Por ejemplo, la Norma UNE-ISO 14064 de 2006, actualizada en 2012. Se trata de una de las normas utilizadas para el cálculo de la huella de carbono corporativa para poder inscribirse en el registro público creado por el Real Decreto 163/2014. Más complejas, por estar basadas en el análisis del ciclo de vida, son las orientadas a la huella de carbono de un producto. La última norma

\footnotetext{
${ }^{1}$ Los sectores difusos, como la agricultura, el transporte, la edificación, el resto de actividades industriales, el comercio, la gestión de residuos etc., independientemente de obligaciones legales que puedan ir surgiendo, se encuentran con la exigencia creciente de los mercados internacionales, en los que los esfuerzos voluntarios en reducir las emisiones de gases de efecto invernadero o huella de carbono son ya una ventaja competitiva.

${ }^{2}$ AENOR es una asociación creada al amparo de lo dispuesto en la Ley de Asociaciones 191/1964, de 24 de diciembre, y del Decreto 1440/1965, de 20 de mayo. Fue designada para el desarrollo de la normalización en el ámbito nacional por Orden del Ministerio de Industria y Energía de 26 de febrero de 1986, de acuerdo con el Real Decreto 1614/1985, y a través del Real Decreto 2200/1995, de 28 de diciembre, por el que se aprueba el Reglamento de la Infraestructura para la Calidad y la Seguridad Industrial, quedó reconocida como organismo de normalización de los establecidos en el capítulo II. E1 Real Decreto 1337/1999, de 31 de julio, por el que se regula la remisión de información en materia de normas y reglamentaciones técnicas y reglamentos relativos a los servicios de la sociedad de información, designó a AENOR como organismo español de normalización, lo que le confiere, entre otras obligaciones, la de informar a la Comisión Europea e intercambiar información sobre los nuevos proyectos de normas nacionales con los organismos de normalización de los demás Estados.
} 
internacional sobre la contabilidad y la información de los GEI de los productos es la Norma ISO 14067, del año 2013. AENOR forma parte de los comités que han dado lugar a esta norma.

Ante la profusión a nivel internacional de estándares y metodologías, se entiende de utilidad para los sectores exportadores de la Región disponer de información avanzada sobre puntos de fundamental importancia como las respuestas a las exigencias de los mercados, los procesos de formación de las normas o las normas internacionales de interés y su aplicación.

La existencia de una delegación de AENOR en la Región de Murcia contribuye a la implantación de la actividad de normalización sobre cambio climático, huella de carbono, ecoeficiencia, ecoinnovación y responsabilidad ambiental para los tejidos económicos y sociales de la región en la Región. Es necesario, por lo tanto, que entre la Administración ambiental y el organismo de normalización se establezca un marco de colaboración por el que se impulse, en el ámbito de nuestra Región, el fomento de la responsabilidad social corporativa aplicada al cambio climático, la economía baja en carbono y la adaptación a los impactos del cambio climático en los términos expresados en la Ley 4/2009, de 14 de mayo, de Protección Ambiental Integrada ${ }^{3}$.

De acuerdo con lo anterior, mediante Resolución de 19 de diciembre de 2014 (BORM, núm. 1, de 2 de enero de 2015) se publica el Convenio de colaboración entre la Comunidad Autónoma de la Región de Murcia, a través de la Consejería de Agricultura y Agua, y AENOR, para impulsar actividades relacionadas con el papel de la normalización en materia de huella de carbono, mitigación y adaptación al cambio climático y promoción de una economía baja en carbono en la Región de Murcia.

\footnotetext{
${ }^{3}$ La Ley 4/2009, de 14 de mayo, de Protección Ambiental Integrada, dedica el título VI al fomento del medio ambiente y la lucha frente al cambio climático. En este sentido, el capítulo I se destina al desarrollo de medidas de fomento de la responsabilidad social corporativa aplicada al medio ambiente, y el capítulo II establece medidas para fomentar una economía baja en carbono y la adaptación a los impactos del cambio climático. Dentro de este último capítulo, el artículo 120 señala que la consejería competente en materia de medio ambiente incentivará la reducción voluntaria de emisiones de gases de efecto invernadero de aquellos sectores de actividad no sometidos a autorización de emisión de gases de efecto invernadero, de conformidad con lo previsto en la Ley $1 / 2005$, de 9 de marzo, por la que se regula el régimen del comercio de derechos de emisión de gases de efecto invernadero. Igualmente, establece que la Consejería competente en materia de medio ambiente impulsará la compensación voluntaria de las emisiones que no hayan podido ser reducidas y fomentará el patrocinio y el mecenazgo en relación con el cambio climático.
} 


\subsection{Zonas de especial protección para las aves (ZEPA)}

\section{A. Lagunas de las Moreras y lagunas de Campotéjar}

Las lagunas de las Moreras y las lagunas de Campotéjar fueron declaradas como zonas de especial protección para las aves (ZEPA) mediante Acuerdo del Consejo de Gobierno de 3 de abril de 2014. Posteriormente, mediante nuevo Acuerdo del Consejo de Gobierno de fecha 12 de diciembre de 2014, dicha declaración se modifica, incorporándose nueva información sobre las especies de aves por las que se han declarado ambos espacios protegidos red Natura 2000 —Lagunas de las Moreras (código ES0000536) y Lagunas de Campotéjar (código ES0000537)—, así como sobre sus límites geográficos conforme a la mejora descriptiva introducida en la relación de coordenadas y en la cartografía de referencia, sin alterarse su delimitación. El nuevo anexo informativo se hizo público mediante Resolución de 18 de diciembre de 2014 del Secretario General de la Consejería de Agricultura y Agua (BORM, núm. 5, de 8 de enero de 2015).

\section{B. Los saladares del Guadalentín}

El pasado 30 de diciembre de 2014, el Consejo de Gobierno, a propuesta de la Consejería de Agricultura y Agua, acordó declarar la ampliación del ámbito territorial de la ZEPA de los Saladares del Guadalentín (código ES0000268). Se modifican sus límites geográficos con la inclusión de las lagunas de Las Salinas de Alhama de Murcia en este espacio protegido de la red Natura 2000, de conformidad con el artículo 44 de la Ley 42/2007, de 13 de diciembre, del Patrimonio Natural y de la Biodiversidad ${ }^{4}$.

De este modo, mediante Resolución de 9 de enero de 2015, del Secretario General de la Consejería de Agricultura y Agua, se dispone la publicación en el BORM del Acuerdo del Consejo de Gobierno de fecha 30 de diciembre de $2014^{5}$. En el anexo que acompaña

\footnotetext{
${ }^{4}$ El artículo 44 de la Ley 42/2007, de 13 de diciembre, del Patrimonio Natural y de la Biodiversidad, bajo la rúbrica "Declaración de las Zonas Especiales de Conservación y las Zonas de Especial Protección para las Aves", dispone que "las Comunidades Autónomas, previo procedimiento de información pública, declararán las Zonas Especiales de Conservación y las Zonas de Especial Protección para las Aves en su ámbito territorial. Dichas declaraciones se publicarán en los respectivos Diarios Oficiales incluyendo información sobre sus límites geográficos, los hábitats y especies por los que se declararon cada uno. De ellas se dará cuenta al Ministerio de Medio Ambiente a efectos de su comunicación a la Comisión Europea, de conformidad con lo establecido en el art. 10 de la Ley 30/1992, de 26 de noviembre, de Régimen Jurídico de las Administraciones Públicas y del Procedimiento Administrativo Común".

${ }^{5}$ La Resolución de 9 de enero de 2015, del Secretario General de la Consejería de Agricultura y Agua, por la que se publica el Acuerdo del Consejo de Gobierno de 30 de diciembre de 2014, relativo a la
} 
al Acuerdo se integra la información sobre las especies de aves por las que se declara y amplía la ZEPA de los Saladares del Guadalentín, así como sobre sus límites geográficos modificados.

\subsection{Estadística}

De conformidad con lo dispuesto en el artículo 149.1.31 ${ }^{\mathrm{a}} \mathrm{CE}$, corresponde al Estado la competencia exclusiva en materia de estadística para fines estatales, la cual recae en el Ministerio de Agricultura, Alimentación y Medio Ambiente. Por otro lado, de conformidad con lo previsto en el artículo 10 de su Estatuto de Autonomía, la Región de Murcia ha asumido la competencia exclusiva sobre las estadísticas para fines de la Comunidad Autónoma. Existe una creciente trayectoria de cooperación en materia de elaboración de estadísticas agroalimentarias entre la Administración general del Estado y la de la Comunidad Autónoma, en el ámbito de sus respectivas competencias. Esta cooperación se materializó mediante la suscripción, con fecha 21 de enero de 2014, de un convenio marco de colaboración en materia de estadística (2014-2016).

Se considera conveniente la armonización de las estadísticas elaboradas por ambas administraciones, así como el establecimiento de una única fuente de recogida de información. Se pretende evitar duplicidades, divergencias y molestias al informante, así como minimizar el coste que supone la ejecución del Programa Estadístico, garantizando, según las exigencias de la normativa nacional y de la Unión Europea, el suficiente grado de cobertura, fiabilidad y oportunidad. Sobre la base de lo anterior, ambas administraciones firmaron un convenio específico de colaboración dirigido a establecer una acción conjunta en materia de estadística agraria, ganadera y de pesca para llevar a cabo, durante el año 2014, las funciones precisas en la recogida de información en explotaciones agrarias, establecimientos, empresas industriales y mercados de productos agrarios, así como su grabación y validación, en el ámbito territorial de la Comunidad Autónoma de la Región de Murcia, para la ejecución del 
Programa de Estadístico. El referido convenio se publicó mediante Resolución de 11 de febrero (BORM, núm. 41, de 19 de febrero de 2015).

\subsection{Biodiversidad}

Con la finalidad de preservar los valores naturales y paisajísticos de nuestra Región y conseguir unos sistemas ecológicamente más valiosos y estables frente a las presiones y agresiones externas a las que puedan estar sometidos, la Comunidad Autónoma de la Región de Murcia lleva a cabo una planificación y gestión activa de estos espacios naturales, los cuales están incluidos en la red Natura 2000 u otras redes ecológicas de protección o figuras similares. Por su parte, la Fundación "la Caixa" destaca entre sus objetivos la mejora de la calidad de vida de los ciudadanos mediante el desarrollo social, cultural y ambiental con la finalidad de lograr un desarrollo humano sostenible. De acuerdo con estos fines, desarrolla un gran número de programas medioambientales y sociales dirigidos a la conservación y mejora del entorno natural, así como a la promoción de la integración social de los colectivos de personas desfavorecidas.

Así pues, dada la existencia de objetivos coincidentes y el interés recíproco de la Comunidad Autónoma de la Región de Murcia y la Fundación "la Caixa” en materia de conservación del medio ambiente, el pasado 15 de diciembre de 2014 ambas partes suscribieron un convenio de colaboración para el desarrollo de actuaciones de conservación de la biodiversidad en la Región de Murcia ${ }^{6}$. El objeto de este no es otro que establecer las condiciones para la cooperación en el desarrollo y la ejecución de las actuaciones para la protección, promoción, conservación y mejora del medio ambiente gestionadas por la Comunidad Autónoma de la Región de Murcia, a través de la Consejería de Agricultura y Agua.. A tal efecto, las partes firmantes se comprometen a impulsar actuaciones medioambientales en la Comunidad Autónoma de la Región de Murcia, siempre dentro del respeto al ámbito competencial de esta ${ }^{7}$. El precitado

\footnotetext{
${ }^{6}$ A tal efecto se dispone de la autorización otorgada por el Consejo de Gobierno en su sesión del 5 de diciembre de 2014. Se tiene en cuenta aquí que tanto el objeto del convenio como las obligaciones establecidas en él regulan un marco de colaboración que concierne al interés público de la Consejería de Agricultura y Agua, a los efectos de lo dispuesto en el artículo 6.6 de la Ley 7/2004, de 28 de diciembre, de Organización y Régimen Jurídico de la Administración Regional.

${ }^{7}$ En relación con las competencias de la Comunidad Autónoma, y de conformidad con el artículo 11.3 del Estatuto de Autonomía para la Región de Murcia, corresponde a la Comunidad Autónoma la competencia de desarrollo legislativo y ejecución en materia de medio ambiente y normas adicionales de protección. Asimismo, ha de tenerse presente que, de conformidad con el Decreto del Presidente núm. 4/2014, de 10 de abril, de reorganización de la Administración Regional, la Consejería de Agricultura y Agua será el
} 
convenio se hizo público mediante Resolución de 11 de febrero de 2015 (BORM, núm. 43, de 21 de febrero de 2015).

\subsection{Ganadería}

La Orden de 16 de septiembre de 1996, por la que se crea el Libro de Registro de Explotación Ganadera, vino a desarrollar el Decreto 205/1996, de 9 de febrero, por el que se establece un sistema de identificación y registro de las especies bovina, porcina, ovina y caprina, y transpone al ordenamiento jurídico español la Directiva 92/102/CEE del Consejo, de 27 de noviembre, relativa a la identificación y registro de animales. Desde ese momento hasta la actualidad se aprecian modificaciones sustanciales en la normativa tanto estatal como comunitaria, de forma que, con el fin de alcanzar en la Región de Murcia una adecuada implantación de los sistemas de identificación y registro de los animales, resulta necesario modificar la Orden de 16 de septiembre de 1996, proporcionando una nueva redacción más acorde con el nuevo marco estatal y comunitario $^{8}$.

A tal fin se dicta la Orden de 12 marzo de 2015, de la Consejería de Agricultura y Agua (BORM, núm. 66, de 1 de marzo de 2015), por la que se modifica la Orden de 16 de septiembre de 1996, de la Consejería de Medio Ambiente, Agricultura y Agua, por la que se crea el Libro de Registro de Explotación Ganadera.

órgano encargado de la propuesta, desarrollo y ejecución de las directrices generales del Consejo de Gobierno en materia de medio ambiente y gestionará el conjunto de zonas o espacios naturales de la Comunidad Autónoma de la Región de Murcia.

${ }^{8}$ Entre las normas que motivan la necesaria modificación de la Orden de 16 de septiembre de 1996, destacan, entre otras: la Ley 8/2003, de 24 de abril, de Sanidad Animal, cuyo artículo 38 dispone que cada explotación de animales deberá mantener actualizado un libro de registro de explotación en el que se registrarán, al menos, los datos que la normativa aplicable disponga, del que será responsable el titular de la explotación; el Real Decreto 804/2011, de 10 de junio, por el que se regula la ordenación zootécnica, sanitaria y de bienestar animal de las explotaciones equinas y se establece el plan sanitario equino, cuyo artículo 6 prevé la existencia de un libro de registro de explotación. A nivel comunitario, el Reglamento (CE) núm. 504/2008 de la Comisión, de 6 de junio de 2008, por el que se aplican las Directivas 90/426/CEE y la 90/427/CEE, se refiere a los métodos de identificación de los equinos y establece un sistema común de identificación y registro de los animales de estas especies. En desarrollo del citado Reglamento (CE) 504/2008, se aprueba el Real Decreto 577/2014, de 4 de julio, por el que se regula la tarjeta de movimiento equina, cuyo artículo 4 establece que los movimientos de salida y retorno a las explotaciones deberán quedar registrados en el libro de registro de explotación. 


\subsection{Agricultura}

A. Vid y cítricos

El Decreto 8/1998, de 26 de febrero, sobre productos agrícolas obtenidos por técnicas de producción integrada, establece en su artículo 8.2 que las normas técnicas de cada cultivo se regularán mediante orden de la Consejería. De acuerdo con lo anterior, diversas órdenes de 24 de abril de 2012 regularon las normas técnicas de producción integrada en los cultivos de vid y cítricos.

Desde entonces han tenido lugar variaciones fundamentales en las materias activas susceptibles de ser aplicadas en el contexto de la producción integrada, lo que hace necesario llevar a cabo una actualización de las normas técnicas. Así, a propuesta de la Dirección General de Industria Agroalimentaria y Capacitación Agraria, y en uso de las atribuciones establecidas en el artículo 8.2 del Decreto 8/1998, de 26 de febrero, sobre productos agrícolas obtenidos por técnicas de producción integrada, y en el artículo 16.2. d) de la Ley 7/2004, de 28 de diciembre, de Organización y Régimen Jurídico de la Administración Pública de la Comunidad Autónoma de la Región de Murcia, la Consejería de Agricultura y Agua, mediante Orden de 24 de marzo de 2015, procede a modificar la Orden de 24 de abril de 2012, de la Consejería de Agricultura y Agua, por la que se regulan las normas técnicas de producción integrada en el cultivo de vid y de cítricos (BORM, núm. 73, de 30 de marzo de 2015).

En concreto, se modifican los anexos III y IV, sobre control fitosanitario y herbicidas permitidos en la producción integrada en vid. Y en relación con las normas técnicas de producción integrada en el cultivo de cítricos, se modifican los anexos IV, V y VI, sobre control fitosanitario en producción integrada en cítricos, productos aplicables en tratamientos post-cosecha de cítricos y herbicidas permitidos en producción integrada de cítricos. 
B. Almendros, cerezos, frutales con hueso, olivos y perales ${ }^{9}$

Como se veía supra, el Decreto 8/1998, de 26 de febrero, sobre productos agrícolas obtenidos por técnicas de producción integrada, establece en su artículo 8.2 que las normas técnicas de cada cultivo se regularán mediante orden de la Consejería. De acuerdo con lo anterior, diversas órdenes de 29 de mayo de 2014, de la Consejería de Agricultura y Agua, fijaron las normas técnicas de producción integrada en el cultivo del almendro, del cerezo, de los frutales con hueso, de los olivos y de los perales.

Dado que durante este tiempo se han producido variaciones en las materias activas susceptibles de ser aplicadas en el contexto de la producción integrada, resultaba necesario llevar a cabo una actualización de dichas normas técnicas. Así pues, a propuesta del director general de Industria Agroalimentaria y Capacitación Agraria, y en uso de las ya examinadas atribuciones establecidas en el artículo 8.2 del Decreto 8/1998, de 26 de febrero, sobre productos agrícolas obtenidos por técnicas de producción integrada, y en el artículo 16.2.d) de la Ley 7/2004, de 28 de diciembre, de Organización y Régimen Jurídico de la Administración Pública de la Comunidad Autónoma de la Región de Murcia, se dicta la Orden de 1 de abril de 2015, de la Consejería de Agricultura y Agua, por la que se modifica la Orden de 29 de mayo de 2014 (BORM, núm. 84, de 14 de abril de 2015).

En concreto, por lo que se refiere al almendro, las modificaciones operadas por la Orden de 1 de abril de 2015 se centran en los anexos III y IV, por los que se regulan, respectivamente, los herbicidas permitidos en la producción integrada en almendro y el control fitosanitario en almendro.

En relación con el cerezo, y al igual que en el caso del almendro, las modificaciones se centran en los anexos III y IV, sobre herbicidas permitidos en producción integrada en cerezo y control fitosanitario en cerezo.

En relación con los frutales de hueso, se modifica el anexo III, destinado a los herbicidas permitidos en producción integrada en frutales de hueso; el anexo IV, sobre

\footnotetext{
${ }^{9}$ La regulación de los almendros, los cerezos, los frutales con hueso, los olivos y los perales se efectúa, en realidad, de forma separada. En este sentido, se identifican cinco órdenes de 1 de abril de 2015, de la Consejería de Agricultura y Agua, por las que se modifican las respectivas órdenes de 29 de mayo de 2014, por las que se regulan las normas técnicas de producción integrada destinadas cada una de ellas de forma específica al cultivo del almendro, del cerezo, de los frutales con hueso, del olivo y del peral. Cada una de estas tiene su propia página en el BORM núm. 84, de 14 de abril de 2015. Si bien, dadas las similitudes existentes y a fin de evitar reiteraciones innecesarias, se considera conveniente su análisis conjunto.
} 
control fitosanitario en albaricoquero; el anexo $\mathrm{V}$, sobre control fitosanitario en melocotonero y nectarino; y, por último, el anexo VI, sobre control fitosanitario en ciruelo.

Por lo que se refiere al olivo, se modifican los anexos siguientes: anexo IV, sobre herbicidas permitidos en producción integrada en olivo; anexo V, sobre control fitosanitario; y anexo VI, relativo al índice de madurez.

Por último, en relación con el peral, se modifican, en línea con los anteriores, los anexos III, IV y $\mathrm{V}$, referidos respectivamente a los herbicidas permitidos en producción integrada, al control fitosanitario en peral y al tratamiento post-cosecha.

C. Derechos de régimen de pago básico y pagos directos a la agricultura y a la ganadería y otros regímenes de ayuda

Nos ocupamos aquí de la Orden de 10 de abril de 2015, de la Consejería de Agricultura y Agua, por la que se regula, en el ámbito de la Región de Murcia, determinados aspectos sobre la asignación de derechos de régimen de pago básico, la aplicación en 2015 de los pagos directos a la agricultura y a la ganadería y otros regímenes de ayuda, las solicitudes de modificación del SIGPAC para los regímenes de ayuda relacionados con la superficie y la presentación de la solicitud única en el marco de la política agrícola común.

Tal y como indica su artículo 1, la Orden tiene por objeto regular, en el ámbito de la Región de Murcia, determinados aspectos sobre la asignación de derechos de pago básico, los requisitos aplicables a los pagos directos a la agricultura y a la ganadería y determinadas ayudas de desarrollo rural, y convocar las ayudas incluidas en la “Solicitud Única” para el año 2015, en el marco de la Política Agrícola Común (PAC) para el período 2015-2020. Se establece el procedimiento para la presentación de la "Solicitud Única", contemplada en el Real Decreto 1075/2014, de 19 de diciembre, sobre la aplicación a partir de 2015 de los pagos directos a la agricultura y a la ganadería y otros regímenes de ayuda, así como sobre la gestión y control de los pagos directos y de los pagos al desarrollo rural. En concreto, esta "Solicitud Única" (solicitud única anual de ayudas) se fija como medio para que cualquier beneficiario pueda presentar su solicitud para todos los regímenes de pagos directos que considere, así como para las ayudas al desarrollo rural financiadas con cargo al Feader. 


\subsection{Urbanismo}

El 6 de mayo de 2015 entró en vigor la Ley 13/2015, de 30 de marzo, de Ordenación Territorial y Urbanística de la Región de Murcia, por la que se deroga en su integridad la Ley del Suelo de la Región de Murcia aprobada por Decreto Legislativo 1/2005, de 10 de junio, quedando derogados asimismo el artículo 111, la disposición adicional primera, la disposición transitoria octava y el anexo IV de la Ley 4/2009, de 14 de mayo, de Protección Ambiental Integrada.

El primer objetivo de la reforma legislativa es el de agilizar los trámites en el ámbito de la ordenación territorial y urbanístico. Se pretende como objetivo primordial que las actividades económicas cuya implantación precise licencias, conforme a otras disposiciones ya vigentes sobre liberalización de servicios, puedan obtenerlas en el menor tiempo posible y sin pérdida de las debidas garantías. En esta línea, destaca como novedad la unificación de los trámites administrativos en materia medioambiental y urbanística, que se llevarán a cabo simultáneamente, con plazos "ciertos y concretos" evitando que los procesos se dilaten en el tiempo. Si bien, este objetivo ha recibido duras críticas por la desprotección ambiental y la potenciación urbanística que puede implicar. También en relación con este objetivo de reducir trámites, se regulan los procedimientos de aprobación de planes de ordenación territorial y urbanística, con una mejor coordinación con los procedimientos ambientales. Con ello se consigue que las cuestiones ambientales estén presentes en la toma de decisiones desde el primer momento y que, además, tanto los documentos necesarios para ello como los trámites precisos se realicen a la misma vez y coordinadamente con los urbanísticos.

La nueva Ley modifica la regulación de la figura de las actuaciones de interés regional (AIR). Se trata de uno de los puntos más controvertidos de la norma en la medida que amplía sus posibilidades de actuación y reduce sus trámites y que se introduce la figura de la comunicación previa o declaración responsable para la autorización de determinadas obras o usos del suelo, como pueden ser las obras menores o la primera ocupación de edificios e instalaciones. Se restringe al máximo la exigencia de licencia municipal de obra y se amplían enormemente los supuestos en los que se puede acudir a la comunicación previa o declaración responsable. Además, se potencian como instrumentos excepcionales las actuaciones de interés regional para aquellas iniciativas cuyas características trasciendan el ámbito municipal y hayan de beneficiar a la Región 
de Murcia en el ámbito de los servicios públicos, la economía, la conservación del medio ambiente o la mejora de la calidad de vida.

En este sentido, no han sido pocas las críticas que toman como punto de partida la Sentencia núm. 428/2013, de 31 de mayo, de la Sala de lo Contencioso-Administrativo del Tribunal Superior de Justicia de Murcia, por la que se resuelve el recurso interpuesto por la Asociación Prolitoral (Ecologistas en Acción, ANSE, Amacora y varios abogados y profesionales del medio ambiente), anulando la Actuación de Interés Regional de Marina de Cope (costa de Águilas y Lorca) por afectar a un espacio protegido que fue desclasificado de manera ilegal. Esta sentencia ha sido recientemente ratificada por el Tribunal Constitucional, que ha declarado inconstitucional la disposición adicional octava de la Ley del Suelo de la Región de Murcia de 2001, por la que se desprotegían 11.500 hectáreas, entre las que se incluía la Marina de Cope, entre otros espacios naturales del litoral regional.

En cuanto a la clasificación del suelo, se precisa que los espacios naturales, aunque en ningún caso puedan ser transformados urbanísticamente, sí se puedan calificar como sistemas generales para su obtención gratuita por parte de los municipios, estableciéndose que la asignación de esta categoría no puede ser arbitraria, sino que debe atender a una motivación adecuada y tener una función estructurante $\mathrm{y}$ determinante de valor justificado, vinculado a un destino claro y determinado por el órgano municipal competente para la aprobación del Plan General. Se trata de una técnica de gestión ligada al cumplimiento de las determinaciones del Plan que pretende la obtención gratuita de suelo de valor ambiental declarado para evitar definitivamente su transformación física y proteger y conservar los valores existentes.

Aun cuando su artículo 1 señala que esta ley tiene por objeto "la regulación de la ordenación del territorio, la ordenación del litoral y de la actividad urbanística en la Región de Murcia para garantizar, en el ámbito de un desarrollo sostenible, el derecho a disfrutar de un medio ambiente adecuado para el desarrollo de la persona y la protección de la naturaleza, el derecho a disfrutar de una vivienda digna y adecuada y la protección del patrimonio cultural y del paisaje", son muchas las críticas que desde los sectores ecologistas se están realizando y cuyo análisis precisa un estudio específico. 


\section{Ordenanzas locales}

\subsection{Alcantarilla: aprobación definitiva de la Ordenanza Municipal de Calidad} Odorífera del Aire (BORM, núm. 93, de 24 de abril de 2015)

El Ayuntamiento de Alcantarilla toma como punto de partida el marco normativo brindado por el artículo $45 \mathrm{CE}$, de acuerdo con el cual "todos tienen derecho a disfrutar de un medio ambiente adecuado para el desarrollo de la persona, así como el deber de conservarlo", y el artículo 4 de la Ley 4/2009, de 14 de mayo, de Protección Ambiental Integrada de la Región de Murcia, en el que se especifica que, para el control de la incidencia ambiental de las actividades, corresponde a las entidades locales "la aprobación de ordenanzas de protección en las materias a que se refiere el párrafo anterior y para regular los emplazamientos, distancias mínimas y demás requisitos exigibles a las actividades que pueden producir riesgos o daños al medio ambiente o la seguridad y salud de las personas".

De acuerdo con esto, el pasado 31 de julio de 2014 se aprobó inicialmente la Ordenanza Municipal de Calidad Odorífera del Aire, cuyo objetivo es regular las medidas necesarias para prevenir y corregir las molestias debidas a olores generados por determinadas actividades e instalaciones en zonas sensibles. Entre las finalidades reconocidas de la norma destacan las de: i) conseguir un alto nivel de protección y un ambiente de calidad en materia olfativa mediante la prevención y la reducción en origen de las emisiones de compuestos susceptibles de generar olores; ii) garantizar los derechos de las personas a la intimidad, su dignidad y el libre desarrollo de su personalidad, su calidad de vida y bienestar, y un medio ambiente adecuado en el marco de un desarrollo sostenible; iii) eliminar los impactos y las molestias debidos a olores procedentes de determinadas actividades que afecten a zonas sensibles.

\subsection{Mazarrón: aprobación inicial de la modificación de la Ordenanza de Uso y} Aprovechamiento de Playas en Mazarrón (BORM, núm. 65, de 20 de marzo de 2015)

El pasado 24 de febrero de 2014 se aprobó, en sesión ordinaria del Pleno del Ayuntamiento, la modificación de la Ordenanza de Uso y Aprovechamiento de Playas en Mazarrón. Así, de conformidad con el artículo 49 de la Ley 7/1985, de 2 de abril, reguladora de las Bases del Régimen Local, dicha aprobación inicial se somete a información pública por plazo de treinta días para que los interesados puedan examinar 
el expediente en la Secretaría del Ayuntamiento y presentar las reclamaciones y sugerencias que estimen pertinentes.

3.3. Lorquí: aprobación inicial de la Ordenanza reguladora del Uso y Vertidos a la Red de Alcantarillado del Municipio de Lorquí (BORM, núm. 67, de 23 de marzo de 2015)

El pasado 12 de marzo de 2015, el Pleno de la Corporación acordó la aprobación inicial de la Ordenanza reguladora del Uso y Vertidos a la Red de Alcantarillado del Municipio de Lorquí. De este modo, y siguiendo los trámites previstos en el artículo 49 de la Ley 7/1985, de 2 de abril, reguladora de las Bases del Régimen Local, se somete el Acuerdo a información pública y audiencia de los interesados para la presentación de reclamaciones y sugerencias por plazo de treinta días.

Cabe referirse, finalmente, a la aprobación definitiva de la modificación de la Ordenanza Municipal reguladora de la Limpieza Viaria y Gestión de Residuos y del Reglamento regulador del uso y vertidos a la red de alcantarillado de Torrepacheco (BORM, núm. 45, de 4 de febrero de 2015). 